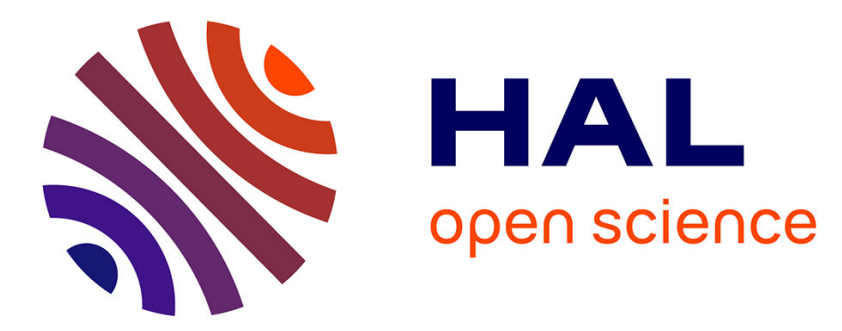

\title{
Power-like decreasing solutions of the linearized Boltzmann equation for neutron thermalization and hard sphere interactions
}

\author{
H. Cornille, A. Gervois
}

\section{- To cite this version:}

H. Cornille, A. Gervois. Power-like decreasing solutions of the linearized Boltzmann equation for neutron thermalization and hard sphere interactions. Journal de Physique Lettres, 1980, 41 (24), pp.581-584. 10.1051/jphyslet:019800041024058100 . jpa-00231851

\section{HAL Id: jpa-00231851 https://hal.science/jpa-00231851}

Submitted on 1 Jan 1980

HAL is a multi-disciplinary open access archive for the deposit and dissemination of scientific research documents, whether they are published or not. The documents may come from teaching and research institutions in France or abroad, or from public or private research centers.
L'archive ouverte pluridisciplinaire HAL, est destinée au dépôt et à la diffusion de documents scientifiques de niveau recherche, publiés ou non, émanant des établissements d'enseignement et de recherche français ou étrangers, des laboratoires publics ou privés. 


\title{
LE JOURNAL DE PHYSIQUE-LETTRES
}

J. Physique - LETTRES 41 (1980) L-581 - L-584

15 DÉCEMBRE 1980, PAGE L-581

Classification

Physics Abstracts

05.60

\section{Power-like decreasing solutions of the linearized Boltzmann equation for neutron thermalization and hard sphere interactions (*)}

\author{
H. Cornille and A. Gervois \\ Service de Physique Théorique, CEN-Saclay, BP nº 2, 91190 Gif-sur-Yvette, France \\ (Reçu le 11 août 1980, acceptéle 31 octobre 1980)
}

\begin{abstract}
Résumé. - Dans le cas neutronique et le cas des sphères dures, on considère les fonctions propres de l'équation de Boltzmann linéarisée correspondant à des valeurs propres discrètes et décroissant comme des puissances inverses de la vitesse. Elles doivent être rejetées parce que ne satisfaisant pas aux conditions physiques de conservation de la masse et de l'énergie.

Abstract. - In the neutronic and hard sphere cases, for non discrete eigenvalues we consider eigenfunctions of the linearized Boltzmann equation decreasing like powers of the velocity. The possible existence of such solutions would imply that there is no minimal relaxation rate to equilibrium. Here we show that they must be rejected because they violate the physical requirement of conservation of mass or energy.
\end{abstract}

The existence or not of solutions of the Boltzmann equation decreasing like inverse powers of energy is very important both for its theoretical and physical implications.

From a theoretical point of view, such solutions lead to a violation of the conventional normalization condition. The relevance to physics of the normalization condition, a well known problem emphasized first by van Kampen [1] and discussed in classical text books like Ford and Uhlenbeck [2] or Waldmann [3], has been a controversial point in the past because the physical arguments for rejecting them were not convincing [2].

Considering the regular spectrum of the linearized Boltzmann equation, the physical consequences of the existence of such solutions appear clearly in simple cases like Maxwell [4] and hard sphere interaction, or neutron thermalization. If they do not exist, there is a minimum relaxation rate; if they do exist, there is a continuous spectrum surimposed to the discrete one, and the relaxation to equilibrium can be arbitrarily slow. It is known that such solutions exist in the linearized Maxwell interaction case [4]; elsewhere [5] we show that they also exist in the full non linear Boltzmann equation. However, as noticed by Hauge and Praestgaard [6], their existence is an open problem in the hard sphere interaction case. Here we investigate

$\left(^{*}\right)$ La version française de cet article a été accepté aux Comptes Rendus de l'Académie des Sciences et elle est insérée dans le $\mathrm{n}^{\circ} \mathrm{du}$ 29 septembre, tome 291, série B, p. 101. both the neutron thermalization and the linearized hard sphere models which present some similarities [7]. We look for the spatially homogeneous and isotropic linearized Boltzmann distribution function and consider non singular eigenfunctions associated with a continuum spectrum with exponential time decay rate and decreasing like the inverse powers of the velocity variable. The result of this paper is the following : solutions exist decaying to equilibrium like $v^{-4}$ ( $v$ being the velocity) in the neutron thermalization model and like $v^{-6}$ in the hard sphere case but they must be rejected from physical requirements because they violate the conservation of mass or the conservation of energy. Let us emphasize that, on the contrary, these physical requirements are fulfilled for the similar power like solutions of the Maxwell gas [5] leading to acceptable new solutions. Consequently Maxwellian and hard sphere interaction lead then to very different physical properties : namely the first one has a minimal relaxation rate and the second one has not.

We begin with the uniform neutron gas with a nearequilibrium velocity distribution

$$
F\left(v^{2}, t\right)=\mathrm{e}^{-v^{2}}+R\left(v^{2}, t\right) .
$$

We consider the eigenfunctions $R\left(v^{2}, t\right)=\mathrm{e}^{-\mu t} R\left(v^{2}\right)$ satisfying the linear equation :

$\left[\left(-\mu+v\left(v^{2}\right)\right)\right] R\left(v^{2}\right)=\int_{0}^{\alpha} K^{\mathrm{N}}(v, w) R\left(w^{2}\right) \mathrm{d} w$ 


$$
\begin{aligned}
K^{\mathrm{N}}(v, w)=\frac{w}{2 v}\left[\mathrm{e}^{-v^{2}+w^{2}} I(w) \theta(v-w)+\right. & \\
& +I(v) \theta(w-v)]
\end{aligned}
$$

where $\theta$ is the step function,

$$
I(v)=\int_{0}^{v} \mathrm{e}^{-u^{2}} \mathrm{~d} u \quad \text { and } \quad v\left(v^{2}\right)=\frac{\mathrm{e}^{-v^{2}}}{8}+\left(\frac{v}{4}+\frac{1}{8 v}\right) I(v)
$$

is the collision frequency. The discrete spectrum of eigenvalues exists for a countable set of

$$
\mu \in\left[0, v(0)=\frac{1}{4}\right] \text {. }
$$

In this letter we consider solutions corresponding to continuum values in the same interval and do not deal with the singular spectrum for $\mu>\frac{1}{4}$. (1)-(2) can be reduced to a second order differential equation

$$
\frac{\mathrm{d}}{\mathrm{d} v}\left\{\frac{\frac{\mathrm{d}}{\mathrm{d} v}\left((\mu-v) v R \mathrm{e}^{v^{2}}\right)}{2 v \mathrm{e}^{v^{2}} I+1}\right\}=\frac{1}{2} v R .
$$

Investigating the asymptotic $(v \rightarrow \infty)$ behaviour of (1) we find that $R$ in general is a linear combination of two solutions behaving asymptotically like $\mathrm{e}^{-v^{2}}$ and $v^{-4}$ and goes to a constant when $v \rightarrow 0$. For $\mu=0$, two independent solutions of the differential equations are

$$
\mathrm{e}^{-v^{2}} \text { and } \mathrm{e}^{-v^{2}} \int_{\text {const. }}^{v} \frac{2 u \mathrm{e}^{u^{2}} I+1}{\left(u v\left(u^{2}\right)\right)^{2}} \mathrm{~d} u .
$$

The last one behaves like $v^{-4}$ when $v \rightarrow \infty$ but is singular like $v^{-1}$ when $v \rightarrow 0$. It follows that for $\mu=0$, solution of (1)-(2) cannot decrease like a power. For $\mu \neq 0$ where the solutions are not known in closed form we would like to study the consistency of a present asymptotic $v^{-4}$ component in $R$ with the physical requirement that the moment

$$
M_{0}=\int_{0}^{\infty} v^{2} R\left(v^{2}, t\right) \mathrm{d} v
$$

must be zero due to the conservation of masses. We integrate both sides of (1)

$$
\begin{aligned}
-\mu \int_{0}^{A} v^{2} R \mathrm{~d} v=\int_{0}^{A} v^{2} & {\left[-v\left(v^{2}\right) R+\right.} \\
& \left.+\int_{0}^{\infty} K^{\mathrm{N}}(v, w) R\left(w^{2}\right) \mathrm{d} w\right] \mathrm{d} v .
\end{aligned}
$$

We notice the identity

$$
\int_{0}^{\infty} v^{2} K^{\mathrm{N}}(v, w) \mathrm{d} v=w^{2} v\left(w^{2}\right) \text { and let } A \rightarrow \infty .
$$

At the 1.h.s. we get $-\mu M_{0}$ whereas at the r.h.s. we have two cases depending upon $R$ contains the $v^{-4}$ component or not. If $v^{-4}$ is not present, both terms at the r.h.s. exist, we can invert the integration on $v$ and $w$ and we find $\mu M_{0}=0$. Otherwise if $v^{-4}$ is present, this procedure is not allowed, we rewrite for finite $A$ the r.h.s. and find

$$
\begin{aligned}
-\frac{\mathrm{e}^{-A^{2}}}{4} \int_{0}^{A} I \mathrm{e}^{v^{2}} v R \mathrm{~d} v+\left(A \mathrm{e}^{-A^{2}}+I(A)\right. & \left.\left(A^{2}-1\right)\right) \times \\
& \times \int_{A}^{\infty} \frac{w R}{4} \mathrm{~d} w .
\end{aligned}
$$

Let us normalize $R$ such that $\lim v^{4} R\left(v^{2}\right)=1$. The first term goes to zero when $A \rightarrow \infty$ whereas the second one gives a non null contribution. We find $-\mu M_{0}=I(\infty) / 8 .(I(\infty)=2 / \sqrt{\pi})$. If $\mu=0$ this result leads to a contradiction and so necessarily $v^{-4}$ cannot be present in $R$, the only solution is the exponential $R=\mathrm{e}^{-v^{2}}$. For $\mu \neq 0$, then $M_{0} \neq 0$ and the corresponding solutions of (1) violate physical requirements.

Another method of study is set up from the analogy with the problem of bound-state in potential scattering where $\mu$ is replaced by imaginary values of the momentum. A solution of the Schrödinger equation, " regular » at the origin, is in general at infinity a linear combination of two solutions : one is decreasing and the other is not bounded. The bound-state condition, leading to discrete eigenvalues, corresponds to the suppression of the unbounded part of the solution. Here let us write an inhomogeneous integral equation, equivalent to (1)-(2) corresponding to regular behaviour condition at $v=0(R(v) \sim 1)$

$$
\begin{aligned}
& \mathrm{e}^{v^{2}} R\left(v^{2}\right)\left(1-\frac{\mu}{v\left(v^{2}\right)}\right)=\left(1-\frac{\mu}{v(0)}\right)-\frac{\mu}{2} \times \\
& \times \int_{0}^{v} w^{2} R\left(w^{2}\right) k(v, w) \mathrm{d} w \\
& k(v, w)=\int_{w}^{v} \frac{2 u \mathrm{e}^{u^{2}} I+1}{\left(u v\left(u^{2}\right)\right)^{2}} \mathrm{~d} u \\
& \mathrm{e}^{-v^{2}} \underset{v \rightarrow \infty}{k(v, w)} \simeq \text { const. } v^{-4} \text {. }
\end{aligned}
$$

A clear investigation of the $v \rightarrow \infty$ behaviour of (3) leads to the conclusion that the only possibility to suppress the $v^{-4}$ term is to require

$$
\int_{0}^{\infty} w^{2} R\left(w^{2}\right) \mathrm{d} w=0 \text { or } M_{0}=0 .
$$

Conversely if $M_{0} \neq 0$ then $R\left(v^{2}\right) \underset{v \rightarrow \infty}{\longrightarrow}$ const. $v^{-4}$.

Another analogy is possible with the Jost function approach of the bound-state problem. Let us write an inhomogeneous integral equation (similar to the Jost solutions) requiring that at infinity $R \simeq \mathrm{e}^{-v^{2}}$ without $v^{-4}$ term 


$$
\begin{array}{r}
R\left(v^{2}\right) \mathrm{e}^{v^{2}}\left(1-\frac{\mu}{v\left(v^{2}\right)}\right)=1+\frac{\mu}{2} \int_{v}^{\infty} w^{2} R\left(w^{2}\right) \times \\
\times k(v, w) \mathrm{d} w,
\end{array}
$$

with $k \rightarrow-\left(v v^{2}(0)\right)^{-1}$ when $v \rightarrow 0$. In order to be a solution of (1)-(2), $R$ must go to a constant when $v \rightarrow 0$ and so we must add that the "Jost function", $\lim v R\left(v^{2}\right)$ is zero or again $\mu M_{0}=0$. Let us notice $0 \rightarrow 0$

that (4) complemented by the condition that the "Jost function" is zero $\left(M_{0}=0\right)$ can be utilized in order to determine the $\mu$ values of the discrete spectrum. In conclusion the condition $M_{0}=0$, generates the discrete spectrum.

Secondly we turn to the hard sphere model where the eigenfunctions satisfy an equation of the equation (1) type with a different kernel $K^{\text {H.S. }}$ :

$$
\begin{aligned}
K^{\mathrm{H} . S .}(v, w)= & 2 K^{\mathrm{N}}-\mathrm{e}^{-v^{2}} \frac{w}{v}\left[\left(\frac{w v^{2}}{2}+\frac{w^{3}}{6}\right) \times\right. \\
& \left.\theta(v-w)+\left(\frac{w^{2} v}{2}+\frac{v^{3}}{6}\right) \theta(w-v)\right]
\end{aligned}
$$

and still investigate the interval $\mu \in\left[0, v(0)=\frac{1}{4}\right]$.

The integral equation (1) with kernel (5) can be reduced to a fourth order differential equation but with the arbitrariness due to an extra polynomial of the second order in $v$ that we can add in (1). This means that all the fundamental solutions of the differential equation are not necessarily present in (1). Investigating the possible asymptotic behaviour of (1) we find that if $M_{0}=0, R$ can be only a linear combination of two components $v^{2}$ et $\mathrm{e}^{-v^{2}}$ and $v^{-6}$ whereas if $M_{0} \neq 0$ there can exist another one, $\mathrm{e}^{-v^{2}}$. Finally the problem is still reduced to searching whether or not the less decreasing component $v^{-6}$ is compatible with the conservation of masses

$$
M_{0}=\int_{0}^{\infty} v^{2} R\left(v^{2}\right) \mathrm{d} v=0
$$

and energy

$$
M_{1}=\int_{0}^{\infty} v^{4} R\left(v^{2}\right) \mathrm{d} v=0 .
$$

As previously we integrate (1)

$$
\begin{aligned}
& -\mu \int_{0}^{A} v^{2 n+2} R \mathrm{~d} v=\int_{0}^{A} v^{2 n+2}\left[-v\left(v^{2}\right) R+\right. \\
& \left.\quad+\int_{0}^{\infty} K^{\mathrm{H.S.}}(v, w) R\left(w^{2}\right) \mathrm{d} w\right] \mathrm{d} v: n=0,1
\end{aligned}
$$

and let $A \rightarrow \infty$. We first consider $M_{0}$ and notice the identity

$$
\int_{0}^{\infty} v^{2} K^{\mathrm{H} . \mathrm{S} .}(v, w) \mathrm{d} v=w^{2} v\left(w^{2}\right) .
$$

In both cases, where $v^{-6}$ is present or not, we can invert the $v, w$ integration variables and we find $\mu M_{0}=0$. It follows that $M_{0}=0$ for $\mu \neq 0$ and at this step an eigenvalue decreasing like $v^{-6}$ is still allowed. Secondly we turn to $M_{1}$ and notice another identity :

$$
\int_{0}^{\infty} v^{4} K^{\text {H.S. }}(v, w) \mathrm{d} v=w^{4} v\left(w^{2}\right) .
$$

If the component $v^{-6}$ is not present we can still invert the $v$ and $w$ integrations and obtain $\mu M_{1}=0$ whereas this is not possible if $v^{-6}$ is present. In this last case we keep $A$ finite, invert integration variables and rewrite the r.h.s.

$$
\begin{aligned}
& \int_{0}^{A} v R\left(v^{2}\right) \int_{0}^{A}\left(\mathrm{e}^{v^{2}} I(v)-\frac{v w^{2}}{2}-\frac{v^{3}}{6}\right) \mathrm{e}^{-w^{2}} w^{3} \mathrm{~d} w \mathrm{~d} v+ \\
& +\int_{A}^{\infty} v R\left(v^{2}\right) \int_{0}^{A} w^{3}\left(I(w)-\mathrm{e}^{-w^{2}}\left(\frac{v^{2} w}{2}+\frac{w^{3}}{6}\right)\right) \mathrm{d} w \mathrm{~d} v .
\end{aligned}
$$

We normalize $R$ in such a way that $\lim _{r \rightarrow r} r^{6} R=1$ and let $A \rightarrow \infty$. The first term goes to zero whereas the second one gives a finite contribution. We find

$$
-\mu M_{1}=\frac{I(\infty)}{16} \neq 0 \text {. }
$$

For $\mu=0$ we have a contradiction which means that a solution $R \simeq v^{-6}$ does not exist for $\mu=0$. On the contrary for $\mu \neq 0$, such solutions may exist, however they yield $M_{1} \neq 0$ and correspond to a violation of the energy conservation law. Conversely if for $\mu \in] 0, \frac{1}{4}\left[\right.$ we require $M_{1}=0, R$ necessarily when $v \rightarrow \infty$ behaves like $v^{2} \mathrm{e}^{-v^{2}}$ and belongs to the discrete spectrum. Finally for the hard sphere model the discretization of the eigenvalue spectrum corresponds to the physical requirement $M_{1}=0$.

Can we extend the above discussion concerning $M_{0}$ (resp. $M_{1}$ ) if the solution of the linear neutronic problem (resp. of the linearized hard sphere case) contains a superposition of such $\mu_{i}$ eigenfunctions ? We restrict the discussion to a countable set of $\mu_{i}$ values : $0<\mu_{0}<\mu_{1} \ldots<\frac{1}{4}$. $M_{0}$ can be written $M_{0}=\gamma_{0} \mathrm{e}^{-\mu_{0} t}+\sum_{i}^{\infty} \gamma_{i} \mathrm{e}^{-\mu_{i} t}$ where $\gamma_{i} \neq 0$ from the above discussion. Clearly, if the number of $\mu_{i}$ is finite, $M_{0}(t)=0 \forall t$ is not allowed. Otherwise, if $\sum\left|\gamma_{i}\right|$ is bounded and $t$ sufficiently large, this sum is, in modulus, less than $\mathrm{e}^{-\mu_{1} t} \sum\left|\gamma_{i}\right|$, then cannot cancel $\gamma_{0} \mathrm{e}^{-\mu_{0 t}}$; consequently, $M_{0}(t) \equiv 0 \forall t$ is not possible.

It may be useful to push a similar analysis for $\mu$ belonging to the singular spectrum $\mu>\frac{1}{4}$, and investigate whether or not the physical requirements of conservation of masses and energy, have a link with the behaviour of the eigensolutions. 


\section{References}

[1] Van Kampen, N. G., Physica 21 (1955) 949.

[2] Uhlenbeck, G. and Ford, G., Lectures in Statistical Mechanics (Proceedings of the Seminar, Boulder Colorado 1960). American Math. Soc. 1963, p. 88.

[3] Waldmann, L., Handb. Phys. 12, 365-367.

[4] Bobylev, A. V., Sov. Phys. Dokl. 20 (1976) 820; Dokl. Akad. Nauk SSR 225 (1975) 1041.

[5] Cornille, H. and Gervois, A., Saclay preprint, to appear in Phys. Lett. A.
[6] Hauge, E. H. and Praestgaard, E., Arkiv. Fys. Semin. Trondheim $\mathrm{n}^{\circ} 11,1979$, ISSW 0365-2459.

[7] Cercignani, C., Theory and Application of the Boltzmann Equation (Scottish Academic Press, Edinburg) 1975. See also : Kuscer, I. and Corngold, N., Phys. Rev. 139A (1965) 981. Kuscer, I. and Williams, M., Phys. Fluids 10 (1967) 1922.

Corngold, N., Michael, P. and Wollman, W., Nucl. Sci. Eng. $15(1963) 13$.

Yan, C. C., Phys. Fluids 12 (1969) 2306.

Jenssen, O., Phys. Norvegica 6 (1972) 179. 